\title{
Virtual image rendering with stationary RGB color correction for mirror image
}

\author{
Jeeva.K,kavithaMargret.M \\ $P G$ Student,Assistant professor,Dept of CSE \\ SVS College of Engineering, Coimbatore, Tamilnadu, India.
}

\begin{abstract}
Digital image processing which concurrently process the stationary images, and RGB colour corrections in our proposed framework.In this paper we introduce two kind of algorithms.The first one is contour algorithm.This algorithm fully based on the background subtraction algorithm.contour algorithm main function is separation of foreground and background of the images.It can capture structural background motion over a long period of time under limited memory using adaptive and compact background model.The second one is edge detection algorithm. Edge detection is a set of mathematical methods used to identifying points in a digital images as well as the image brightness changes sharply.In this algorithm edges are termed by set of curved line segments.This edges are very useful to change the brightness of the images.The input image foreground is merged with different background using these two kind of algorithms in our proposed method.The separate foreground images are enhanced by using depth analysis method.The depth analysis method is used to find the distance between foreground and background of the images.
\end{abstract}

\section{Introduction}

Any form the signal processing in imaging science is called as an image processing.In image processing we are giving the input is an image that will The knoor some parameters related to that given image.The digital image processing refers to image processing. Multidimentional signals are manipulated by the modern digital image processing techniques. Photography is the most common type in image processing. Analogue or the digital image is created by cameras.

An image is scan or captured by the camera. The image is stored as a computer file in digital photography.Using the photographic software, this file is translated to an actual image. More complex algorithms are used by the digital image processing.Image is converted into digital form for processing the image.

Quantization of sampled values and sampling of images are include in the digitization.For the image processing the image is converted into the bit information.This image processing is also called as an image enhancement.Today the image processing is growing technologies. It also used in various application areas such as business and medical field.The image processing is used various research areas like engineering and computer science field.The following 5 groups are the purpose of image processing visualization,image restoration and sharpening,image retrevial.

There are two types of imge processing.The first one is analogue image processing, and another is digital imge processing.Some fundamental In Digital Image Processing.

1.Image Acquisition

2.Image pre processing

3.Image segmentation

4.Image Representation and Description

5.Image Recognition and inter

6.knowledge base

In the digital image processing, the image aquistion is the first step process.The digital image is acquired by using this image acquisition process. The main function of image pre processing is to improve the image quality and also deals with removing the noise.For the specific application, image enhancement is more suitable than than original image.

Image preprocessing contain some functions. They are image enhancement, image restoration, image segmentation.The raw pixel data is the output of the segmentation.The image recognition is used for assigns a label to an object based on the information provided by its descriptors. The knowledge database is in the form of knowledge about a problem domain is coded into an image processing system. The knowledge base is guiding the process of each module and also control interaction between the module.

With the help of the digital image technique the computers are create the digital images.This paper using artificial neural networks to providing a new approach for recognize the blur and mirror images.Using the 
digital image processing a framework is developed.This framework is process the stationary images and RGB color correction at the same time.

Two types of inputs is used by this framework, the first one is laptop based and another one is computer based.The images are recognized by the artificial neural networks. The color of the images is recognized by the support vector machine.The support vector machine is gained from the field of pattern classification and machine learning. The separating function can be expressed in support vector classification as a linear combination of kernels.

The kernels are using the support vector.This paper the contour algorithm is proposed.The foreground and background of the image is separated by using this contour algorithm. This algorithm allow the users to multiple changing background.

During the initial training period, unconstrained training that allows moving foreground objects in the scene.For finding the edges on the images the edge detection method is used.The shapes and charecters are recognized by the edge detection method.The edge detection method is used for separate the foreground and background of the image. The set of mathematical method is called as an edge detection method.Points in the digital image is identified by using the edge detection method. And edge dection is the fundamental tool in the field of feature extraction and feature detection methods. The edge detection method is shown in the fig 1.
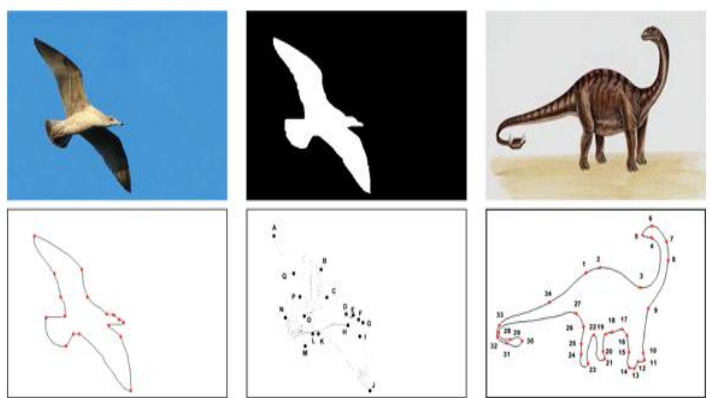

FIGURE 1:Edge detection method

After separating the foreground and background image the depth analysis look for the new background.The images are matched by the depth analysis. The distance between the foreground and background is called depth analysis. The depth analysis relates to film and photography depth of field is the distance between the nearest and farthest objects in a scene that appear acceptably sharp in an image.

A large depth of field is often called deep focus in cinemaphotography and a small depth of field is often called shallow focus. A new foreground image and background image while fusing will get a new fused image with new background, this process is called fuse images. The vital spatial domain fusion method is the high pass filtering based technique.For analysing the remote sensing images the multi resolution analysis has become very useful tool. For the fusion the discrete wavelet transform has become a very useful tool. These tools show a better performance in spatial and spectral quality of the fused image compared to other spatial methods of fusion. The depth analysis is shown in the figure 2.

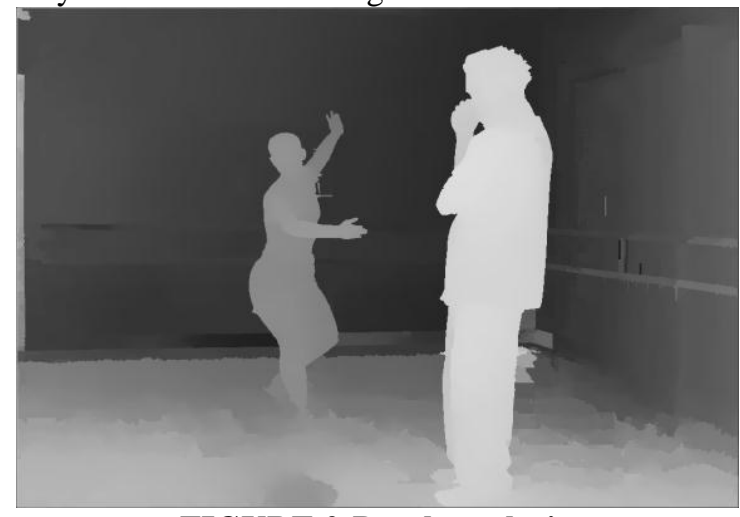

FIGURE 2:Depth analysis

\section{SIMULATION}

\section{Virtual Mirror Modelling And}

The mirror of physical model is very straightforward.In a virtual system there are three main components are used for the process. Three components in virtual mirror modelling and considerations are:

1.The environment structure in 3-D and color.

2.Pose of the mirror and 3-D location. 
3.The view point of the pupil of the eye is more precisely coordinated to 3-D.Here we limited to a single eye in a full stereoscopic system we duplicate the rendering process for each eye and the results are displayed stereoscopic.

The mirror image observation is determined by the viewpoint location and but not the pose of the viewer.The eye ball pose is not relevant to the mirror image,we not track the viewer pose but we need to track the position.

The Kinect depth measurements is noisy.The absorption quality is caused by the missing and erroneous depth values and also the poor reflection or even shadow refection of the light patterns.In IR camera some background regions are visible.In this regions the depth values can not be measured.A novel stochastic framework is used to separates the depth image into foreground and background.

\section{III.DEPTH DENOISING AND COMPLETION}

Two major types of errors in depth images. They are missing and distorted depth values.Fig 3(a) and 3(b) represents pair of typical RGB and depth images. The fig 3(c) show the virtual view of RGB-D system errors.Denoising and completing depth pixeks is based on foreground or background separation in our proposed algorithm.

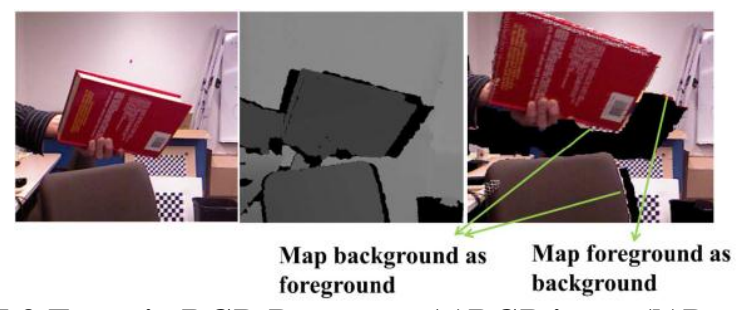

FIGURE 3:Error in RGB-D systems:(a)RGB image(b)Depth image

Two stages are performed in foreground and background separetions. The first one is offline data collections and second one is online segmentation. The static background for training and pre-storage collected by offline stage.Each pixel as either foreground or background labeled by our online algorithm.

\section{Architecture View}

The contour algorithm and the edge detection methods are used to separate the foreground and background of the input image.These two algorithms are perform the separation process in efficient manner.The inputs are videos or any images. If the input is taken as videos, it will be converted to frames.After converting the videos into frames,separation of the foreground and background process is done by the contour algorithm.

Edge detection method also used to separate the foreground and background of the images.Edge of the image is identified by the edge detection method.Depth information is collected,after the foreground and background separation of images.The foreground image is separated from the background image.The depth of the image is analysed by the depth analysis process. The separated foreground image is enhanced by the depth analysis method. An appropriate background image is merged with enhanced foreground image.The fused image will provide the new image with high quality. The architecture view is demonstrate in the figure 4.

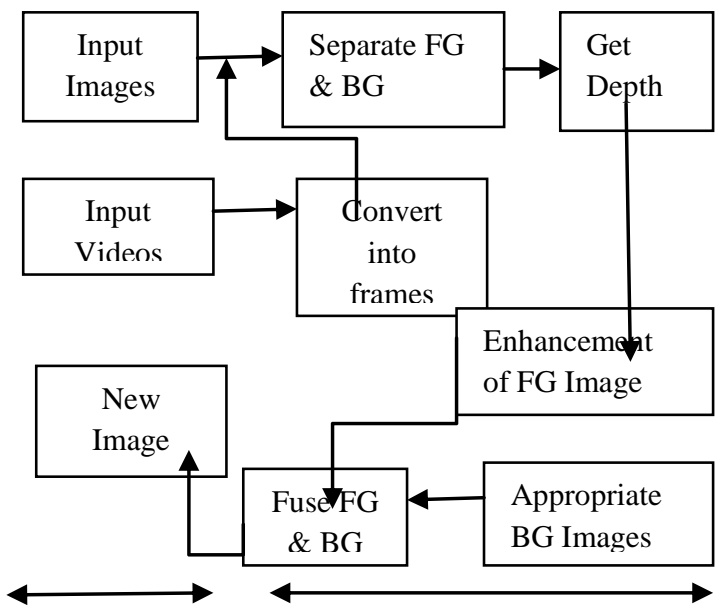

New FG \& BG Merging Original FG image with FIGURE 4:Architexture overview 


\section{System Integration And Mirror Image Synthesis}

The mirror rendering system implemented by four main components. The following four components are mainly used to implement the mirror rendering system.

1.Background scanning

2.Depth denoising and completion

3.Data acquisition

4.Final view synthesis

An offline process that builds the 3-D model of the background using background scanning.The RGBD camera and client computer are connected by using the Depth denoising and completion,data acquisition and mirror view generation modules.The synthesis final images are displayed by final view synthesis module.Background scanning component is udes to scan the background of the images.Both depth denoising and completion algorithms are used to remove the noise from the images. These contour and edge detection algorithms are fully based on foreground and background separation.

\section{Countour Algorithm}

The proposed contour algorithm is used to separate the foreground and background of the images.Elevation and altitude,depth of the images are denoted by the contour algorithm.In cartography the most common usage is the countour line.The contour algorithm have The capability of coping with local and global illumination changes. The following algorithm is contour algorithm.

I.L $\leftarrow \mathbf{0}^{\mathbf{1}}, \mathbf{I} \leftarrow \emptyset$ (empty set)

II. For $\mathbf{t}=\mathbf{1}$ to $\mathbf{N}$ do

$$
\text { i. } X_{t}=(\mathbf{R}, \mathbf{G}, \mathbf{B}), \mathbf{I} \leftarrow \sqrt{R^{2}+G^{2}+B^{2}} \quad \text { ii. Find the codeword } \mathbf{C}_{\mathbf{m}} \text { in } \mathbf{I}=\left\{\mathbf{C}_{\mathbf{i}} \mid \mathbf{1} \leq \mathbf{i} \leq \mathbf{L}\right\} \text { matching }
$$

to $\mathbf{X}_{\mathbf{t}}$ based on two conditions (a) and (b).

a) Colordist $\left(\mathbf{X}_{\mathbf{t}}, \mathbf{V}_{\mathbf{m}}\right) \leq \boldsymbol{\varepsilon}_{\mathbf{1}}$

b) Brightness $\left(\mathbf{I}\left\langle\breve{\mathbf{I}}_{\mathrm{m}}, \hat{\mathbf{I}}_{\mathrm{m}}\right\rangle\right)=$ true

iii.sIf $\mathbf{l}=\emptyset$ or there is no match, then

iv. $\mathbf{L} \leftarrow \mathbf{L}+\mathbf{1}$.create a new codeword $\mathbf{C}_{\mathbf{L}}$ by setting

- $\mathbf{V}_{\mathbf{L}} \leftarrow(\mathbf{R}, \mathbf{G}, \mathbf{B})$

- $\operatorname{aux}_{\mathbf{L}} \leftarrow\langle\mathbf{I}, \mathbf{I}, 1, \mathrm{t}-1, \mathrm{t}, \mathrm{t}\rangle$

III. Otherwise, update the matched codeword $\mathbf{C}_{\mathbf{m}}$, consisting of

$$
\mathbf{V}_{\mathrm{m}}=\overline{\boldsymbol{R}}_{\mathrm{m}}, \overline{\boldsymbol{G}}_{\mathrm{m}} \overline{\boldsymbol{B}}_{\mathrm{m}} \text { and } \mathbf{a u x}_{\mathrm{m}}=\breve{\mathbf{I}}_{\mathrm{m}}, \hat{\mathbf{I}}_{\mathrm{m}}, \mathbf{f}_{\mathrm{m}}, \lambda_{\mathrm{m}}, \mathbf{p}_{\mathrm{m}}, \mathbf{q}_{\mathrm{m}}>\text { by setting }
$$$$
\mathbf{V}_{\mathbf{m}} \leftarrow\left(\frac{f_{m} \bar{R}_{m}+R}{f_{m}+1}, \frac{f_{m} \bar{G}_{m}+G}{f_{m}+1}, \frac{f_{m \bar{B}_{m}+B}}{f_{m}+1}\right)
$$

$\left.\operatorname{aux}_{\mathrm{m}} \leftarrow<\min \left\{\mathbf{I}, \breve{\mathbf{I}}_{\mathrm{m}}\right\}, \max \left\{\mathbf{I}, \hat{\mathbf{I}}_{\mathrm{m}}\right\}, \mathbf{f}_{\mathrm{m}}+\mathbf{1}, \max \left\{\boldsymbol{\lambda}_{\mathrm{m}}, \mathbf{t}-\mathbf{q}_{\mathrm{m}}\right\}, \mathbf{p}_{\mathrm{m}}, \mathbf{t}\right\rangle$

end for

IV.Each codeword $\mathbf{C}_{\mathbf{i}}, \mathbf{I}=\mathbf{1}, \ldots \ldots ., \mathbf{L}$, wrap around $\lambda_{\mathbf{i}}$ by setting $\lambda_{\mathbf{I}} \leftarrow \max \left\{\lambda_{\mathbf{i}},\left(\mathbf{N}-\mathbf{q}_{\mathbf{i}}+\mathbf{p}-\mathbf{1}\right)\right\}$

The following parameters are used in the contour algorithm.L-length,t-nodes,l-subset of L,X-foreground

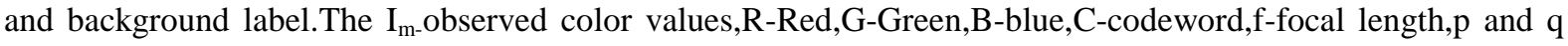
denote the closest points.

\section{Experimental Results}

The first experimental evaluation is separation of foreground and background for thin objects using contour algorithm. This algorithm take raw RGB frame as an input.The depth of the input frame estimated by using the depth analysis as well as it produce raw depth image. The foreground mask is the further step in this experiment. Finally our raw RGB image merge with new background. First simulation phase take input image using open function of the panel. 


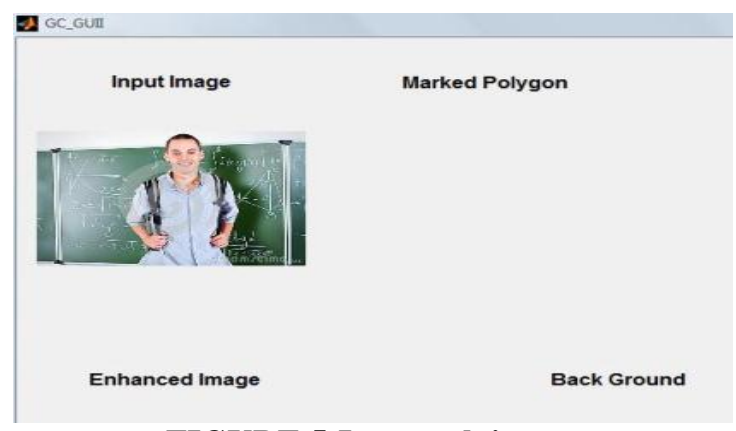

FIGURE 5:Input rgb image

The second simulation phase is marked polygon. This polygon is marked by using mark polygon button in the panel.Fig 6(a) represents the marked polygon area.The selected area is represented by the red color line.The marked image is the foreground image.The foreground image is enhanced for better quality result for the simulation.For the foreground and background separation the selected area is only considered.

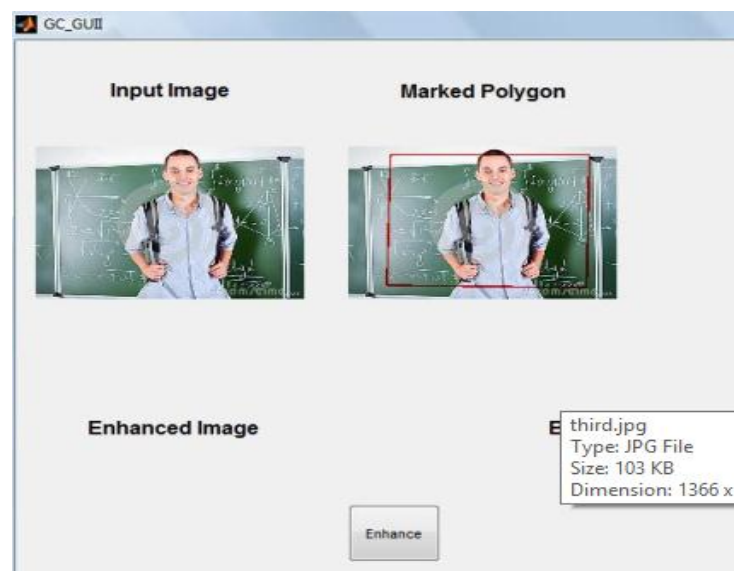

\section{FIGURE 6(a):Marked polygon}

The panel consist four kind of functions.They are open,marked polygon,load polygon and run.Fig 6(b) represents the panel frame.The load polygon option used for separate foreground of the image.This separation of foreground updated on current result as well as it display the acknowledgement.Fig 7(a) and (b) denotes the current result and acknowledge of the current result respectively.This foreground separation done by our proposed algorithm.

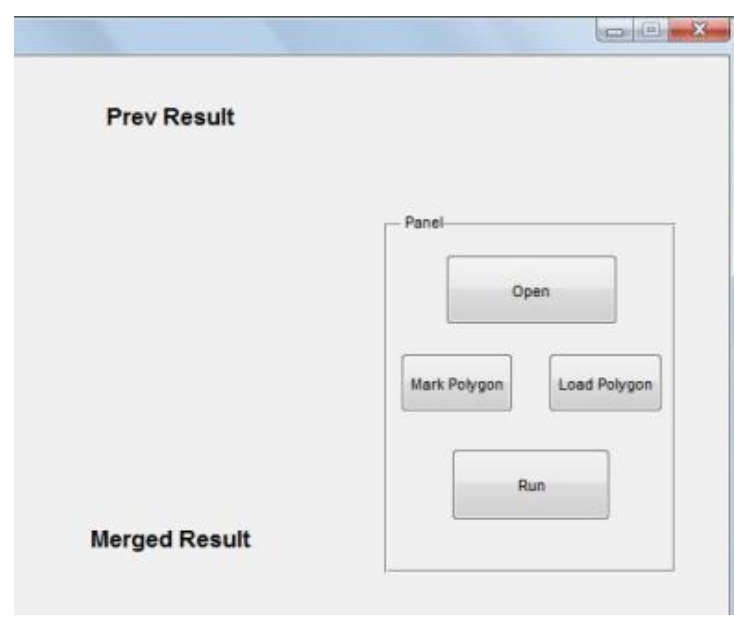

FIGURE 6(b):panel frame

The final simulation of run function provide the merging foreground result.In the current result image is foreground image.This image is separated from background image 


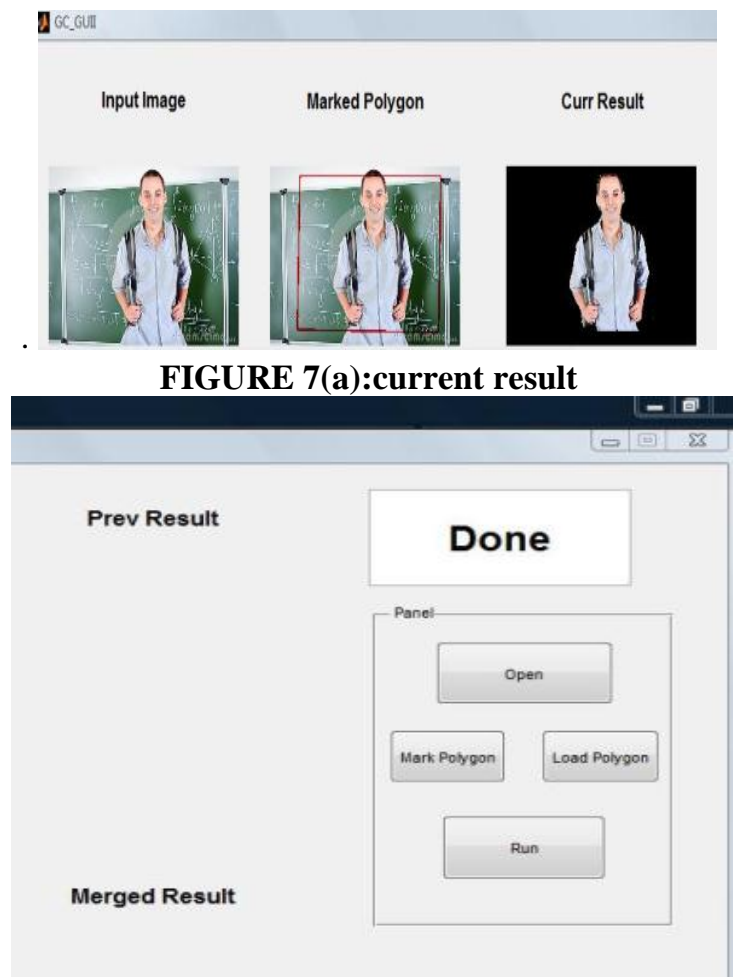

FIGURE 7(b):current result acknowledgement

The enhance function used to enhance the foreground image using depth analysis method.The current result efficiently enhanced by background selection. The below figure shows the enhanced image.

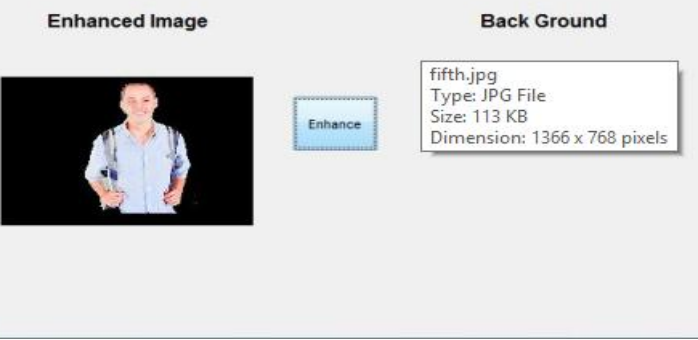

The selection of background proceed successfully by corresponding function.we introduce two types of parameters like $\mathrm{K}$ and Beta.The parameter $\mathrm{K}$ take the charge of image pixels. The parameter represents the brightness of image.These two parameters are estimated by using edge detection algorithm and also parameters are adjustable. The fig. 8 demonstrate background selection

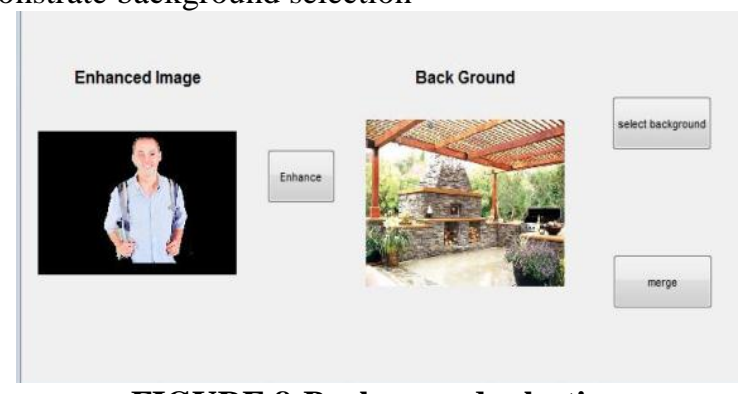

FIGURE 8:Background selection

VIII. Conclusion

Edge detection plays an vital role in image processing and analysis. The main scope of this project is to develop a framework for digital image processing which concurrently process the stationary images and RGB colour corrections.The contour algorithm separate foreground and background of images in efficient manner. The foreground image is enhanced by using the depth analysis method.The foreground image merged with new background image.Our proposed scheme provide efficient result and also improve the quality of the images.Our 
proposed edge detection algorithm fully based on the background subtraction algorithm. The following figure display the experimental simulation result.

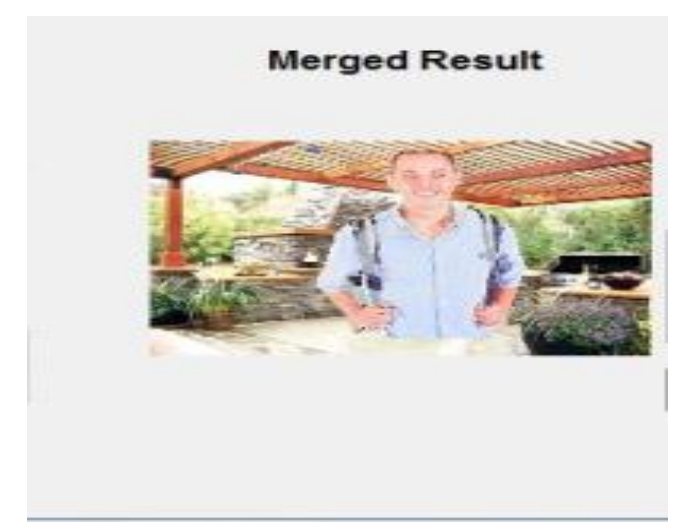

\section{References}

[1] P. Eisert, P. Fechteler, and J. Rurainsky, "3-D tracking of shoes for virtual mirror applications," in Proc. Int. Conf. Comput. Vis. Pattern Recognit. Jun. 2008, pp. 1-6.

[2] J. Shen, S-C S. Cheung, and J. Zhao, "Virtual mirror by fusing multiple RGB-D cameras," in Proc. APSIPA Ann. Summit Conf., Dec. 2012,pp. 1-9.

[3] C. Eisenacher, Q. Meyer, and C. Loop, "Real-time view-dependent rendering of parametric surfaces," in Proc. Symp. Interact. 3-D Graph.Games, 2009, pp. 137-143.

[4] P. Debevec, Y. Yu, and G. Boshokov, "Efficient view-dependent image based rendering with projective texture-mapping," EECS Dept., Univ.California at Berkeley, Berkeley, CA, USA, Tech. Rep. UCB/CSD-98-1003, Jun. 1998.

[5] V. Kitanovski and E. Izquierdo, "3-D tracking of facial features for augmented reality applications," in Proc. Int. Workshop Image Anal. Multimedia Interact. Services, vol. 2. Apr. 2011.

[6] T. Shao, W. Xu, K. Zhou, J. Wang, D. Li, and B. Guo, "An interactive approach to semantic modeling of indoor scenes with an rgbd camera,"ACM Trans. Graph., vol. 31, no. 6, pp. 136-147, Nov. 2012

[7] Q. Zhang, M. Ye, R. Yang, Y. Matsushita, B. Wilburn, and H. Yu, "Edgepreserving photometric stereo via depth fusion," in Proc. IEEE CVPR,Jun. 2012, pp. 2472-2479.

[8] L. Wang, R. Villamil, S. Samarasekera, and R. Kumar, "Magic mirror:A virtual handbag shopping system," in Proc. IEEE Comput. Soc. Conf. CVPRW, Jun. 2012, pp. pp. 19-24.

[9] V. Garro, C. dal Mutto, P. Zanuttigh, and G. M. Cortelazzo, "A novel interpolation scheme for range data with side information," in Proc. IEEE Vis. Media Prod. Conf., Nov. 2009, pp. 52-60.

[10] R. Newcombe, S. Izadi, O. Hilliges, D. Molyneaux, D. Kim,A. J. Davison, P. Kohli, J. Shotton, S. Hodges, and A. Fitzgibbon,"KinectFusion: Real-time dense surface mapping and tracking," inProc. 10th IEEE Int. Symp. Mixed Augmented Reality, Oct. 2011,pp. 127-136 